INGENIERIA AMBIENTAL

\title{
Aplicación del proceso Fenton en el tratamiento de aguas residuales de origen petroquímico
}

ENVIRONMENTAL ENGINEERING

\section{Application of Fenton process for treating petrochemical wastewater}

\author{
Ainhoa Rubio-Clemente * $§$, Edwin L. Chica **, Gustavo A. Peñuela * \\ * Grupo de Diagnóstico y Control de la Contaminación "GDCON”, Universidad de Antioquia, Medellín, \\ Colombia. **Departamento de Ingeniería Mecánica. Universidad de Antioquia, Medellín, Colombia. \\ §ainhoarubioclem@gmail.com,echica@udea.edu.co,gustavo.penuela413@gmail.com
}

Recibido: 30 de Julio de 2013 - Aceptado: 20 de Junio de 2014

\begin{abstract}
Resumen
Este artículo hace una revisión sobre las aplicaciones del proceso Fenton para el tratamiento de aguas residuales procedentes de la industria petroquímica. En él se describe brevemente el mecanismo de reacción y se analizan las principales variables que influyen en la eficiencia de este proceso (concentración del agente oxidante y del catalizador, temperatura, $\mathrm{pH}$, tiempo de reacción, tipo de contaminante y presencia de aniones). Asimismo, se detallan diversos estudios que evalúan la viabilidad del sistema Fenton en la degradación de sustancias derivadas del petróleo, sus limitaciones y las perspectivas de futuro.
\end{abstract}

Palabras clave: Aguas residuales, Industria petroquímica, Proceso Fenton, Procesos avanzados de oxidación.

\begin{abstract}
This article reviews the application of Fenton process to treat wastewater from the petrochemical industry. The reaction mechanism is briefly described, and the main variables that influence the efficiency of this process are analyzed (oxidizing agent and catalyst level, temperature, $\mathrm{pH}$, reaction time, type of pollutant and the presence of anions). Additionally, several studies evaluating the feasibility of the Fenton system in the degradation of oil-derived substances are described, as well as Fenton process' limitations and future perspectives.
\end{abstract}

Keywords: Wastewater, Petrochemical industry, Fenton process, Advanced oxidation processes. 


\section{Introducción}

Las aguas residuales provenientes de la industria petroquímica contienen una gran cantidad de compuestos orgánicos de alto potencial contaminante, entre los que destacan fenoles, éteres e hidrocarburos, tales como benceno, tolueno, xileno y poliaromáticos, entre otros. A pesar de que la presencia de estas sustancias en las aguas residuales es variable, la mayor parte de ellas están catalogadas por la Agencia de Protección Ambiental de Estados Unidos y por la Unión Europea como sustancias prioritarias a tratar y eliminar de las aguas, dadas sus características carcinogénicas, mutagénicas y teratogénicas (Hasan et al., 2012; Oller et al., 2011).

En rasgos generales, los contaminantes presentes en las aguas residuales de origen petroquímico presentan baja solubilidad en el agua, son lipofílicos y se bioacumulan a lo largo de la cadena trófica, representando una seria amenaza para los ecosistemas (Rubio-Clemente et al., 2014; Hasan et al., 2012). Dadas sus características y propiedades físico-químicas particulares, en ocasiones, cada uno de estos compuestos requiere de un tratamiento específico para su eliminación efectiva. Si bien, suelen presentarse formando mezclas complejas, de modo que se dificulta su remoción de las aguas. Además, son sustancias extremadamente resistentes y no pueden ser degradadas por las plantas de tratamiento que operan con sistemas convencionales (Dopar et al., 2011, Manoli \& Samara, 2008). Por ejemplo, Manoli \& Samara (2008) encontraron que la unidad de cloración con la que operaba una planta de tratamiento convencional era capaz de transformar como máximo un 10\% de los poliaromáticos presentes en el afluente. Además, aunque se observaron porcentajes de remoción de hasta un $67 \%$ en el tratamiento primario (desarenador-desengrasador seguido de un sedimentador primario), no se evidenció degradación de los mismos, dada la gran afinidad de estas sustancias, tales como el benzo(a)pireno, para adsorberse sobre el material particulado separado (Qiao et al., 2014). Por otro lado, cabe recordar que los procesos convencionales de tipo biológico usados para el tratamiento de aguas residuales petroquímicas dependen fuertemente de los constituyentes presentes y de sus concentraciones (Hasan et al., 2012); de modo que, cuando éstos en grandes cantidades, pueden inhibir el tratamiento biológico. De hecho, los efluentes originados en las refinerías de petróleo presentan altos niveles de compuestos aromáticos y alifáticos, los cuales pueden ser extremadamente tóxicos para los microorganismos encargados del proceso de degradación biológica (Dopar et al., 2011). Por todo ello, se hace necesario el estudio de la viabilidad de otros métodos que pudiesen degradar este tipo de contaminantes (Hasan et al., 2012; Homem et al., 2009).

En este sentido, uno de los tratamientos alternativos y/o complementarios a los sistemas convencionales son los procesos avanzados de oxidación (PAO). Estas tecnologías fueron definidas por Glaze et al. (1987) como aquellos procesos que implican la generación de radicales hidroxilo $\left({ }^{\circ} \mathrm{OH}\right)$ en cantidades suficientes como para degradar la materia orgánica del medio. Estos radicales, de alto potencial de oxidación $\left(\mathrm{E}^{\circ}=2.80 \mathrm{~V}\right)$, pueden ser generados por diversos métodos (combinación de ozono/peróxido de hidrógeno, procesos Fenton y foto-Fenton, procesos fotocatalíticos heterogéneos, etc.) y son capaces de oxidar compuestos orgánicos persistentes, posibilitando su completa mineralización y transformación a $\mathrm{CO}_{2}, \mathrm{H}_{2} \mathrm{O}$ y otros compuestos inorgánicos estables (Bianco et al., 2011; Ghosh et al., 2010). Por otro lado, diversos estudios demuestran que los PAO pueden ser utilizados para tratar contaminantes a muy bajas concentraciones ([pireno $]=49 \mathrm{nM}$ (Manariotis et al., 2011), [nitrobenceno] $=1.2 \mu \mathrm{M}$ (Nichela et al., 2013)) y, por lo general, no generan grandes volúmenes de lodos que requieran de un complejo proceso de tratamiento posterior $(0.24$ $\mathrm{g} / \mathrm{L}$ para 3 -cianopiridina y $0.32 \mathrm{~g} / \mathrm{L}$ para piridina (Padoley et al., 2011)).

En las últimas décadas, los PAO han demostrado ser bastante eficientes en el tratamiento de aguas tanto potables como residuales respecto de los tratamientos de tipo convencional (Babuponnusami \& Muthukumar, 2014; Mandal et al., 2010; Grčić et al., 2009). Sin embargo, estas tecnologías presentan limitaciones en el tratamiento de aguas 
residuales a escala industrial debido a sus altos costos operacionales, en particular, por el consumo energético que requieren y por el costo de los reactivos (agentes oxidantes y/o catalizadores) a utilizar (Babuponnusami \& Muthukumar, 2014; Pontes et al., 2010). Para la reducción del gasto energético, se utilizan PAO compatibles con el uso de la energía solar, tales como el proceso fotoFenton solar. Otra de las alternativas para disminuir los costos y posibilitar la degradación efectiva de la carga orgánica total, así como su toxicidad, consiste en la combinación de PAO con procesos de tipo biológico (Babuponnusami \& Muthukumar, 2014; Oller et al., 2011; Mandal et al., 2010).

En la literatura existen numerosas publicaciones sobre el uso de PAO para la remoción de contaminantes de las aguas residuales. Dentro de estas publicaciones se destacan las relacionadas con el proceso de oxidación tipo Fenton por su efectividad para la descontaminación de aguas residuales procedentes de la industria petroquímica, textil, farmacéutica, papelera, etc. (Babuponnusami \& Muthukumar, 2014; Bianco et al., 2011; Ghosh et al., 2010; Coelho et al., 2006). Empleando los procesos Fenton se puede obtener una degradación parcial o total de contaminantes inorgánicos y orgánicos persistentes, dando lugar a una disminución de la toxicidad del efluente (DuránMoreno et al., 2011). El propósito de este artículo consiste en discutir el mecanismo de reacción y las variables más influyentes en el proceso de oxidación Fenton aplicado al tratamiento de aguas residuales de origen petroquímico.

\section{Proceso Fenton}

El proceso de oxidación Fenton fue descrito por primera vez en 1894 por Henry J. Fenton, cuando descubrió que el peróxido de hidrógeno $\left(\mathrm{H}_{2} \mathrm{O}_{2}\right)$ podía activarse con iones ferrosos para oxidar ácido tartárico (Pignatello et al., 2006). Este proceso consiste en la adición de sales de hierro en presencia de $\mathrm{H}_{2} \mathrm{O}_{2}$, en medio ácido, para la formación de radicales ${ }^{\circ} \mathrm{OH}$. A la combinación de $\mathrm{H}_{2} \mathrm{O}_{2}$ y sales de hierro se denomina reactivo Fenton (Ec. 1 y 2) (Ghosh et al., 2010; Neyens \& Baeyens, 2003).

$$
\begin{gathered}
\mathrm{Fe}^{2+}+\mathrm{H}_{2} \mathrm{O}_{2} \rightarrow \mathrm{Fe}^{3+}+\mathrm{OH}^{-}+\circ \mathrm{OH} \\
\mathrm{Fe}^{3+}+\mathrm{H}_{2} \mathrm{O}_{2} \rightarrow \mathrm{Fe}^{2+}+\mathrm{H}^{+}+\mathrm{HO}_{2} 。
\end{gathered}
$$

Además de formarse radicales ${ }^{\circ} \mathrm{OH}$, se generan radicales perhidroxilo $\left(\mathrm{HO}_{2}^{\circ}\right)$, los cuales inician una reacción de oxidación en cadena para eliminar la materia oxidable. Sin embargo, los radicales $\mathrm{HO}_{2}^{\circ}$ presentan menor poder de oxidación que los ${ }^{\circ} \mathrm{OH}$ (Domenech et al., 2004). Los principales mecanismos de reacción que se llevan a cabo con estos últimos son abstracción de hidrógeno, adición a la estructura del contaminante o transferencia de carga (Ec. 3, 4 y 5, respectivamente). En la Figura 1 se muestra una posible ruta de degradación a partir del proceso Fenton del fenol (Zazo et al., 2005) y del metil ter-butil éter (Hong et al., 2007; Xu et al., 2004), dos de los principales contaminantes de efluentes petroquímicos. Puede observarse que el proceso de transformación del contaminante inicia con la adición del radical ${ }^{\circ} \mathrm{OH}$ a su estructura (Fig. 1a) o con la abstracción de un átomo de hidrógeno (Fig. 1b), dando lugar a intermediarios de reacción que a su vez pueden seguir oxidándose hasta $\mathrm{CO}_{2} \mathrm{y} \mathrm{H}_{2} \mathrm{O}$.

$$
\begin{gathered}
\mathrm{RH}+{ }^{\circ} \mathrm{OH} \rightarrow \mathrm{R}^{\circ}+\mathrm{H}_{2} \mathrm{O} \\
\mathrm{RH}+{ }^{\circ} \mathrm{OH} \rightarrow \mathrm{RHOH}^{\circ} \\
\mathrm{RH}+{ }^{\circ} \mathrm{OH} \rightarrow \mathrm{R}^{\circ+}+\mathrm{HO}^{-}
\end{gathered}
$$

Por su parte, los iones férricos $\left(\mathrm{Fe}^{3+}\right)$ resultantes (Ec. 1) pueden, asimismo, reaccionar con el $\mathrm{H}_{2} \mathrm{O}_{2}$, lo cual conduce a la regeneración del $\mathrm{Fe}^{2+}$ (Ec. 2) (Ghosh et al., 2010). La constante de velocidad de esta reacción tiene un valor de 0.01 $\mathrm{M}^{-1} \mathrm{~s}^{-1}$, aproximadamente (Neyens \& Baeyens, 2003), por lo que se considera como la reacción limitante del proceso.

Además, durante el sistema Fenton puede tener lugar el consumo de radicales ${ }^{\circ} \mathrm{OH}$ (Pignatello et al., 2006; Domenech et al., 2004). Un exceso de $\mathrm{H}_{2} \mathrm{O}_{2}$ y/o de iones de hierro al inicio del proceso, respecto de la cantidad de contaminante a tratar, limita la eficiencia del mismo (Ec. 6 y 7). De igual modo, puede producirse la recombinación de radicales ${ }^{\circ} \mathrm{OH}$ y/o la reacción de éstos con radicales $\mathrm{HO}_{2}^{\circ}$, traduciéndose en la reducción del 
a)

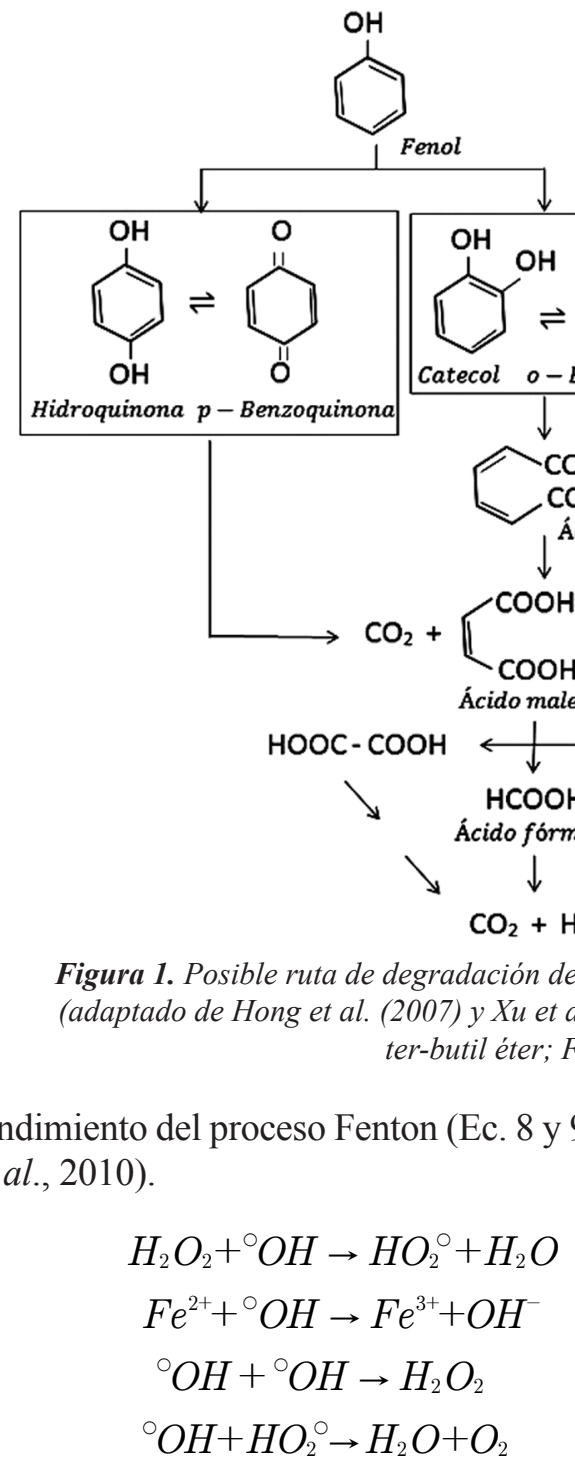

El hierro puede ser añadido en su forma ferrosa $\left(\mathrm{Fe}^{2+}\right)$ o férrica $\left(\mathrm{Fe}^{3+}\right)$, siendo ésta última conocida como proceso tipo Fenton. Las sales de hierro férricas suelen ser menos utilizadas como consecuencia de la lenta generación de radicales ${ }^{\circ} \mathrm{OH}$ en las fases iniciales del proceso, ya que éste se iniciaría con la reacción 2 . Sin embargo, cuando se combina con radiación ultravioleta (UV) o solar, en cuyo caso recibe el nombre de proceso fotoFenton UV y foto-Fenton solar, respectivamente, su eficiencia aumenta significativamente (Mandal et al., 2010), ya que se favorece la regeneración del $\mathrm{Fe}^{2+}$ (Ec. 10), y aumenta, al mismo tiempo, el número de radicales ${ }^{\circ} \mathrm{OH}$ generados (Ec. b)

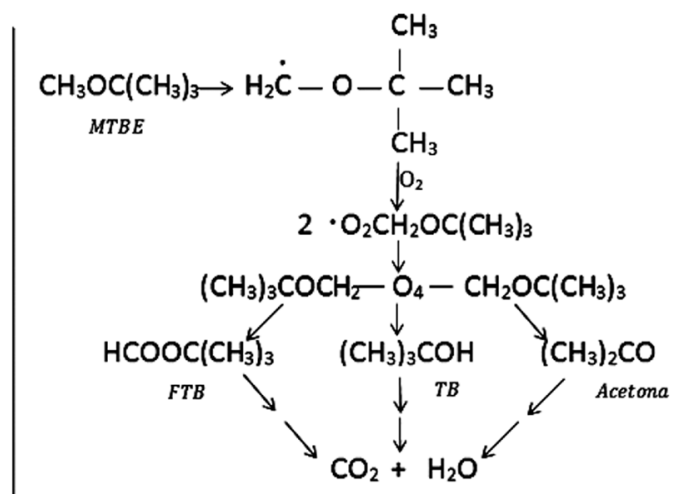

10) (Pignatello et al., 2006). Este hecho puede explicarse a partir de la formación a $\mathrm{pH}$ ácido de complejos acuosos de $\mathrm{Fe}^{3+}\left(\mathrm{Fe}(\mathrm{OH})^{2+}\right)$, los cuales son capaces de absorber radiación UV y visible (Kavitha \& Palanivelu, 2004), sufriendo fotoreducción y produciendo ${ }^{\circ} \mathrm{OH}$ (Batista \& Pupo, 2012; Pignatello et al., 2006).

$$
\mathrm{Fe}^{3+}+\mathrm{H}_{2} \mathrm{O}+h v \rightarrow \mathrm{Fe}^{2+}+\mathrm{H}^{+}+{ }^{\circ} \mathrm{OH}
$$

\subsection{Parámetros que influyen en el proceso}

Diversos autores coinciden en que el rendimiento del proceso Fenton depende, entre otros factores, de la concentración del agente oxidante y catalítico, temperatura, $\mathrm{pH}$ y tiempo de reacción (Pontes et al., 2010; Ghosh et al., 2010; Neyens \& Baeyens, 2003). Asimismo, la eficiencia de este proceso está relacionada con la naturaleza del contaminante a degradar y con la presencia de otros compuestos orgánicos e inorgánicos (Pignatello et al., 2006; Kavitha \& Palanivelu, 2004). En la Tabla 1 se recoge un breve resumen de las condiciones de 
Tabla 1. Aplicación de PAO para el tratamiento de contaminantes presentes en las aguas residuales de la industria petroquímica.

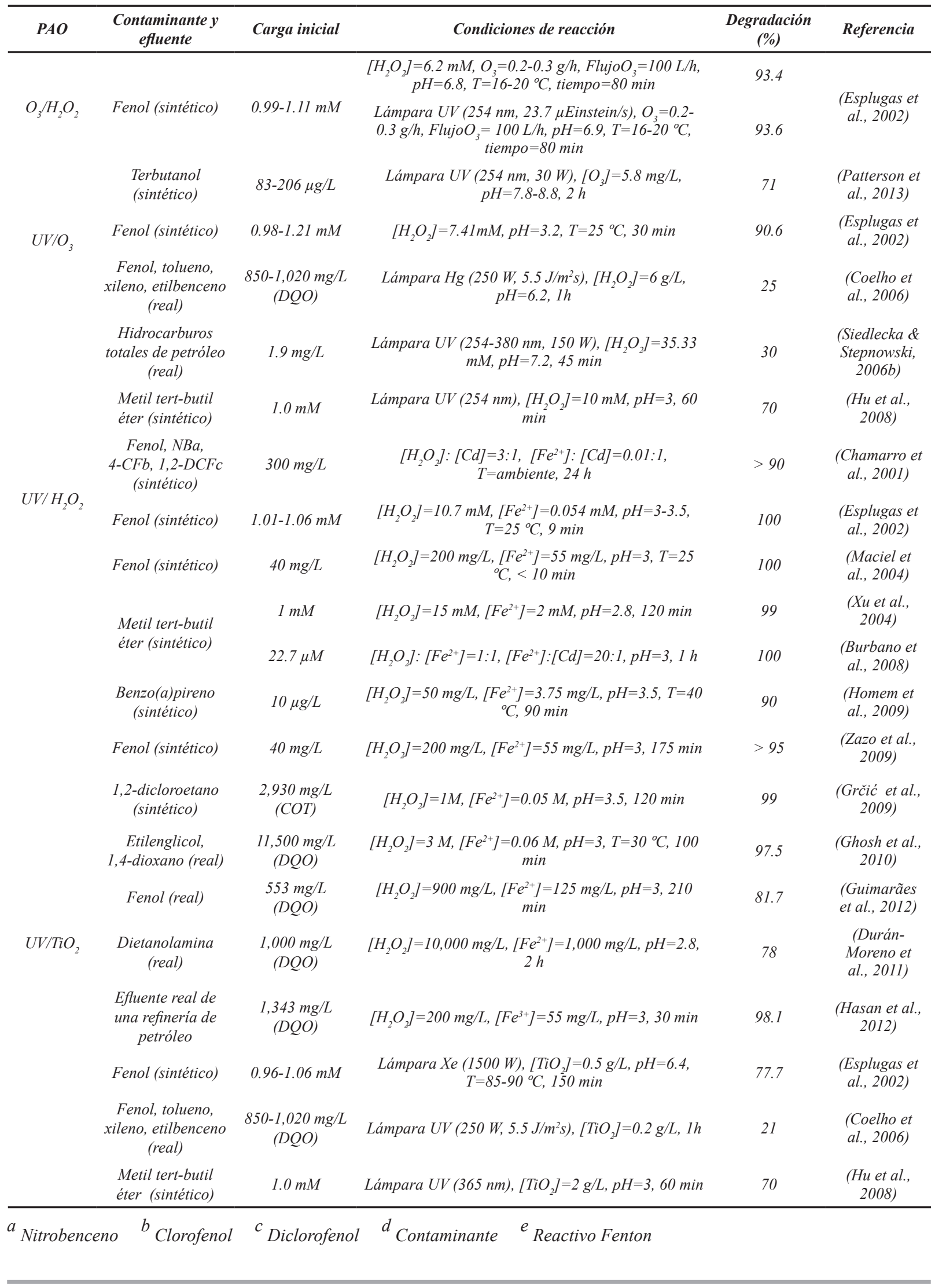


reacción y porcentajes de degradación de diferentes trabajos realizados sobre la aplicación del proceso Fenton en el tratamiento de los principales contaminantes presentes en las aguas residuales petroquímicas. A su vez, en esta tabla se destaca la eficiencia del sistema Fenton en la remoción de dichos contaminantes respecto de otros PAO $\left(\mathrm{O}_{3} /\right.$ $\mathrm{H}_{2} \mathrm{O}_{2}, \mathrm{UV} / \mathrm{O}_{3}, \mathrm{UV} / \mathrm{H}_{2} \mathrm{O}_{2}$ y UV/TiO 2 ).

\subsubsection{Influencia de la concentración del agente oxidante y del catalizador}

La cantidad de $\mathrm{H}_{2} \mathrm{O}_{2}$ y de sales de hierro a utilizar depende del tipo de contaminante que se pretenda remover. Cabe destacar que el $\mathrm{H}_{2} \mathrm{O}_{2}$, requiere de grandes cantidades de $\mathrm{Fe}^{2+}$ para su descomposición en radicales ${ }^{\circ} \mathrm{OH}$. Es de esperar que la velocidad de degradación de las moléculas orgánicas sea mayor a medida que aumenta la concentración del oxidante y del catalizador; sin embargo, grandes cantidades de $\mathrm{H}_{2} \mathrm{O}_{2}$ y de $\mathrm{Fe}^{2+}$ limitan el porcentaje de degradación del contaminante, ya que se favorecen las reacciones secundarias (Ec. 6-9) (Neyens \& Baeyens, 2003). Por ejemplo, Homem et al. (2009) encontraron que un aumento en la concentración de $\mathrm{H}_{2} \mathrm{O}_{2}$ no suponía una mayor degradación de benzo(a) pireno $(10 \mu \mathrm{g} / \mathrm{L})$; ya que con una concentración inicial de $\mathrm{H}_{2} \mathrm{O}_{2}$ de $50 \mathrm{mg} / \mathrm{L}$ se conseguía una degradación del 90\% tras 90 min de reacción; mientras que una concentración de $150 \mathrm{mg} / \mathrm{L}$ sólo permitía eliminar el $80 \%$ del contaminante. Por su parte, Ghosh et al. (2010) observaron una reducción máxima de DQO $(11,500 \mathrm{mg} / \mathrm{L})$ del $97.5 \%$ para una concentración de $\mathrm{Fe}^{2+}$ de $0.06 \mathrm{M}$ en un tiempo de tratamiento de $100 \mathrm{~min}$; niveles de $\mathrm{Fe}^{2+}$ de $0.1 \mathrm{M}$ llevaban a la reducción en la eficiencia del proceso, con una remoción de DQO del $80 \%$.

Existen diversas experiencias en las que se reportan los valores idóneos de agente oxidante y catalítico (Tabla 1). Sin embargo, para determinar las concentraciones óptimas de estas variables es necesario conocer el tipo de contaminante presente en el agua objeto de análisis, al igual que su concentración. Por este motivo, es necesario llevar a cabo la caracterización previa de cada tipo de agua residual a tratar.

\subsubsection{Influencia de la temperatura}

A pesar de que el proceso Fenton puede ocurrir a temperatura ambiente, un aumento de ésta favorece la cinética de las reacciones de oxidación. Homem et al. (2009) manifestaron la viabilidad de operar a altas temperaturas (alrededor de $70{ }^{\circ} \mathrm{C}$ ), consiguiendo remociones de benzo(a)pireno $(10 \mu \mathrm{g} / \mathrm{L})$ del 90 y $100 \%$ a 30 y $70{ }^{\circ} \mathrm{C}$, respectivamente. No obstante, el incremento de temperatura puede disminuir la estabilidad del $\mathrm{H}_{2} \mathrm{O}_{2}$, ya que altos valores de temperatura aumentan la velocidad de degradación del $\mathrm{H}_{2} \mathrm{O}_{2}$ a $\mathrm{O}_{2}$ y $\mathrm{H}_{2} \mathrm{O}$ (Malíková et al., 2009).

\subsubsection{Influencia del pH}

El $\mathrm{pH}$ es una de las variables más significativas en el proceso Fenton. Estudios sobre el reactivo Fenton han demostrado que sólo es efectivo para un rango de $\mathrm{pH}$ que oscila entre 2.5-4; si bien algunos autores (Durán-Moreno et al., 2011; Pignatello et al., 2006; Xu et al., 2004) fijan este valor a 2.8 , siendo este $\mathrm{pH}$ el óptimo para la formación de radicales ${ }^{\circ} \mathrm{OH}$ a partir de la descomposición del $\mathrm{H}_{2} \mathrm{O}_{2}$ en presencia de $\mathrm{Fe}^{2+}$. Condiciones de $\mathrm{pH}$ muy ácidas o muy alcalinas dan lugar a un descenso en la velocidad de degradación de los contaminantes. Un aumento del $\mathrm{pH}$ conduce a la precipitación del hierro como $\mathrm{Fe}(\mathrm{OH})_{3}$, impidiendo que se lleve a cabo la reacción $2 \mathrm{y}$, por tanto, la regeneración de $\mathrm{Fe}^{2+}$. Por otro lado, a pH excesivamente ácido se forma $\left[\mathrm{Fe}\left(\mathrm{H}_{2} \mathrm{O}\right)_{6}\right]^{2+}$, el cual reacciona lentamente con el $\mathrm{H}_{2} \mathrm{O}_{2}$, ralentizando la generación de radicales ${ }^{\circ} \mathrm{OH}$ (Pignatello et al., 2006). A su vez, bajo estas condiciones de acidez, la regeneración del catalizador a partir de $\mathrm{H}_{2} \mathrm{O}_{2}$ se ve inhibida, ya que a un bajo $\mathrm{pH} \mathrm{el} \mathrm{H}_{2} \mathrm{O}_{2}$ se estabiliza en forma de iones oxonio $\left(\mathrm{H}_{3} \mathrm{O}_{2}^{+}\right)$(Malíková et al., 2009; Pignatello et al., 2006). Ghosh et al. (2010) estudiaron el efecto del $\mathrm{pH}$ en el tratamiento de un efluente con $11,500 \mathrm{mg} / \mathrm{L}$ de DQO bajo condiciones óptimas de temperatura $\left(30^{\circ} \mathrm{C}\right), \mathrm{H}_{2} \mathrm{O}_{2}(3 \mathrm{M}), \mathrm{Fe}^{2+}(0.06$ $\mathrm{M})$ y para un tiempo de reacción de $100 \mathrm{~min}$. Los resultados muestran una conversión de DQO de $\sim 85,97.5$ y $65 \%$ a pH 2,3 y 5 , respectivamente. 


\subsubsection{Influencia del tiempo de reacción}

Desde un punto de vista económico, se ha demostrado que operar con altos tiempos de residencia no es viable (Ghosh et al., 2010); de hecho, con bajos períodos de reacción se consiguen buenos niveles de degradación de contaminantes en comparación con otros procesos de oxidación química (Burbano et al., 2008; Esplugas et al., 2002). Esplugas et al. (2002) evaluaron el rendimiento de distintos $\mathrm{PAO}$ para la degradación de fenol (Tabla 1), destacándose el proceso Fenton por su efectividad en la remoción de la totalidad del fenol después de 9 min de tratamiento.

\subsubsection{Influencia del tipo de contaminante}

Las aguas residuales petroquímicas se distinguen por su alto contenido en demanda química de oxígeno (DQO) y en carbono orgánico total (COT). A título orientativo, se reportan valores de DQO de 11,500 mg/L (Ghosh et al., 2010) y 13,068 mg/L (Durán-Moreno et al., 2011), y de COT de 2,930 mg/L (Dopar et al., 2011) y 5,500 $\mathrm{mg} / \mathrm{L}$ (Grčić et al., 2009). Tanto la DQO como el COT son parámetros esenciales a la hora de conocer el grado de contaminación de las aguas (Hasan et al., 2012) y de gran importancia en la eficiencia del proceso Fenton (Bianco et al., 2011). A altos niveles de contaminantes, aumenta el número de colisiones entre éstos y el catalizador y los radicales ${ }^{\circ} \mathrm{OH}$, lo cual posibilita y acelera la degradación de los compuestos tóxicos (Bianco et al., 2011). Sin embargo, una cantidad excesiva de contaminantes en las aguas podría reducir dichas colisiones, ralentizando el proceso de reacción. Estudios realizados por Ghosh et al. (2010) reportaron una disminución en la constante de velocidad de reacción $(\mathrm{k})$ de aproximadamente el $14 \%$ al tratar bajo las mismas condiciones dos efluentes petroquímicos de distinta DQO $(3,100$ $\mathrm{mg} / \mathrm{L}(\mathrm{k}=0.064 / \mathrm{min})$ y $5,950 \mathrm{mg} / \mathrm{L}(\mathrm{k}=0.055 / \mathrm{min})$ ). La reducción en la velocidad de degradación del efluente de origen puede deberse a la formación de subproductos de reacción (Hong et al., 2007). Cuanto mayor es la cantidad inicial de contaminantes, se generará un mayor número de intermediarios, los cuales a su vez reaccionarán con radicales ${ }^{\circ} \mathrm{OH}$, oxidándose y dejando un menor número de ${ }^{\circ} \mathrm{OH}$ disponibles para interactuar con las sustancias de origen (Hong et al., 2007). Además, la naturaleza del contaminante es de especial interés en el rendimiento del proceso, pues el carácter recalcitrante de una sustancia puede ser indicativo de la necesidad de una mayor cantidad de oxidante, la cual debe de incrementarse si dicho contaminante está presente en altos niveles. A su vez, las sustancias tóxicas pueden participar en el proceso de regeneración del $\mathrm{Fe}^{2+}$ (Ec. 11) (Bautista et al., 2007).

$$
\mathrm{Fe}^{3+}+\mathrm{R}^{\circ} \rightarrow \mathrm{Fe}^{2+}+\mathrm{R}^{+}
$$

Por otro lado, debe destacarse que, a pesar de que el reactivo Fenton suele ser considerado como un potente agente oxidante, éste puede no ser completamente efectivo para algunas especies químicas como ácidos carboxílicos de cadena corta (ácido málico, acético, etc.) (Bianco et al., 2011). Este aspecto resulta de especial interés, particularmente porque dichas sustancias, con frecuencia, forman parte de los subproductos generados durante las reacciones de oxidación; los cuales, a su vez, pueden ser considerados como nutrientes para los microorganismos (Oller et al., 2011). Así, parte de los subproductos procedentes del proceso Fenton puede ser biodegradable, mejorándose de este modo la biodegradabilidad del afluente a tratar (Chamarro et al., 2001). Si bien, al mismo tiempo, puede suceder que los subproductos formados sean tóxicos para la comunidad microbiana, lo cual también debe ser tenido en cuenta, especialmente cuando se emplea el proceso Fenton como pretratamiento a un sistema biológico (Oller et al., 2011).

\subsubsection{Influencia de la presencia de aniones}

Determinados aniones, tanto orgánicos como inorgánicos, participan en la formación de complejos de hierro, impidiendo que éste siga reaccionando. La formación de tales compuestos depende, en gran medida, del $\mathrm{pH}$, e influye directamente en la cantidad de iones férricos y ferrosos libres en el agua, restringiendo el ciclo catalítico del proceso Fenton (Malíková et al., 2009). Esta limitación puede ser superada usando radiación UV o solar, ya que permite la degradación de tales complejos, 
como sucede con los ferrioxalatos (Litter \& Quici, 2010; Dopar et al., 2010). Por otro lado, se ha reportado que la presencia de bicarbonatos y carbonatos, fosfatos, sulfatos y cloruros en el agua ocasiona una disminución de la reactividad del sistema Fenton (Levchuk et al., 2014). A modo de ejemplo, los cloruros actúan como secuestradores de radicales ${ }^{\circ} \mathrm{OH}$, inhibiendo la reacción de los mismos (Levchuk et al., 2014). Siedlecka \& Stepnowski (2006a) evaluaron la influencia de iones cloruros a bajas concentraciones (0.05-1 $\mathrm{M})$ en la degradación de metil ter-butil éter $(0.5$ $\mathrm{mM})$; los resultados muestran una reducción en la transformación de este compuesto del 90 al 60\%, aproximadamente.

\section{Aplicaciones del proceso Fenton para la remoción de contaminantes de la industria petroquímica. Limitaciones y perspectivas futuras}

Existen numerosos reportes que aprovechan los beneficios del proceso Fenton (Tabla 2) para el tratamiento de aguas residuales industriales con altas cargas de compuestos tóxicos y resistentes a los tratamientos convencionales (Bianco et al., 2011; Durán-Moreno et al., 2011; Ghosh et al., 2010; Grčić et al., 2009). Experiencias como las realizadas por Homem et al. (2009), Burbano et al. (2008) y Esplugas et al. (2002) prueban la viabilidad de este sistema para conseguir la completa degradación de compuestos orgánicos, como el benzo(a)pireno, el metil ter-butil éter y el fenol, respectivamente. No obstante, este tipo de tecnologías presentan limitaciones (Tabla 2), haciendo necesaria la adopción de otro tipo de alternativas de tratamiento más eficientes, tales como los procesos de oxidación foto-Fenton. Diversos estudios realizados hasta la fecha demuestran este hecho (da Rocha et al., 2013; Coelho et al., 2006; Galvão et al., 2006; Kavitha \& Palanivelu, 2004). Coelho et al. (2006) analizaron la influencia de diversos PAO $\left(\mathrm{UV} / \mathrm{H}_{2} \mathrm{O}_{2}, \mathrm{UV} /\right.$ $\mathrm{TiO}_{2}$, Fenton y foto-Fenton) en el tratamiento de un agua residual procedente de una refinería de petróleo ( $\mathrm{DQO}=820-1,020 \mathrm{mg} / \mathrm{L})$. Todos los procesos estudiados dieron lugar a resultados poco satisfactorios, con remociones $<30 \%$ (Tabla 1), a excepción de las tecnologías Fenton y foto-Fenton. No obstante, el proceso foto-Fenton resultó ser el más efectivo en el tratamiento de este tipo de afluentes, al conseguir una remoción de DQO del $83 \%$. Asimismo, Galvão et al. (2006) probaron la eficiencia del proceso de oxidación foto-Fenton. Trabajaron con un agua residual contaminada con diésel a la que trataron con $0.1 \mathrm{mM}$ de $\mathrm{Fe}^{2+}, 50$ $\mathrm{mM}$ de $\mathrm{H}_{2} \mathrm{O}_{2}$ y radiación UV (lámpara de mercurio de media presión, $450 \mathrm{~W}$ ), consiguiendo una degradación de dicho contaminante superior al

Tabla 2. Ventajas e inconvenientes del proceso de oxidación Fenton.

Ventajas
Gran aplicabilidad. Se ha empleado con éxito en la
degradación de contaminantes presentes en efluentes
petroquímicos (Tabla 1) y puede ser utilizado para el
tratamiento de fangos y suelos contaminados (Pignatello et
al., 2006; Neyens \& Baeyens, 2003).

Los reactivos utilizados son abundantes, comercialmente asequibles y de fácil manejo (Levchuk et al., 2014, Dopar et al., 2011; Bautista et al., 2007).

No existen limitaciones en la transferencia de masa (Litter \& Quici, 2010) y los reactores utilizados son sencillos de operar (Rubio-Clemente et al., 2014).

No precisa de fuente de energía para producir radicales ${ }^{\circ}$ OH y las reacciones de oxidación pueden llevarse a cabo en condiciones de presión y temperatura ambiente (Levchuk et al., 2014; Bautista et al., 2007).

\section{Inconvenientes}

En ocasiones, no se consigue la mineralización completa del contaminante (Burbano et al., 2008, Maciel et al., 2004).

El hierro y $\mathrm{H}_{2} \mathrm{O}_{2}$ residual pueden contaminar el medio receptor (Levchuk et al., 2014; Babuponnusami \& Muthukumar, 2014).

Requiere de un ajuste de $\mathrm{pH}$ antes y después del tratamiento, así como de un tratamiento posterior para eliminar el hierro residual en el efluente (Levchuk et al., 2014; Bautista et al., 2007).

Formación de complejos de hierro estables (Dopar et al., 2011).

\section{8}


99\%. Ambos estudios (Coelho et al., 2006; Galvão et al., 2006) demuestran que la radiación UV combinada con el proceso Fenton permite obtener una remoción apreciable de la carga orgánica. Si bien, el empleo de lámparas UV artificiales hace incrementar los costos de operación (Litter \& Quici, 2010). Esta limitación puede ser superada mediante el empleo de radiación solar (Litter \& Quici, 2010). Por ejemplo, Kavitha \& Palanivelu (2004) evaluaron el rendimiento del proceso fotoFenton para la degradación de un afluente que contenía $2.12 \mathrm{mM}$ de fenol. Las condiciones de trabajo fueron: $\left[\mathrm{H}_{2} \mathrm{O}_{2}\right]=30 \mathrm{mM},\left[\mathrm{Fe}^{2+}\right]=0.8 \mathrm{mM}$, $\mathrm{pH}=3, \mathrm{~T}=$ ambiente y radiación solar. Tras $10 \mathrm{~min}$ de reacción se alcanzaron remociones del orden del $90 \%$. Otra forma de abaratar costos es aprovechar el contenido de hierro presente en las aguas a tratar (da Rocha et al., 2013). Da Rocha et al. (2013) trabajaron con efluentes procedentes de actividades extractivas del petróleo caracterizadas por la presencia de metales, como el Fe, $\mathrm{Cu}, \mathrm{Mn}, \mathrm{Cd}$ y $\mathrm{Zn}$, y un contenido de hidrocarburos aromáticos policíclicos (HAP) de $1.6 \mathrm{mg} / \mathrm{L}$. Los resultados de este estudio después de $7 \mathrm{~h}$ de exposición a la radiación solar y usando $485.3 \mathrm{mM}$ de $\mathrm{H}_{2} \mathrm{O}_{2}$ y 0.93 $\mathrm{mg} / \mathrm{L}$ de hierro, mostraron una eliminación de HAP del $93 \%$.

En comparación con el sistema Fenton, el proceso foto-Fenton permite la formación de una mayor cantidad de radicales ${ }^{\circ} \mathrm{OH}$ y la regeneración de $\mathrm{Fe}^{2+}$ (Ec. 10), permite aprovechar la capacidad fotolítica de los complejos de hierro formados, tales como los ferrioxalatos (Dopar et al., 2011). Sin embargo, el proceso foto-Fenton, al igual que el proceso Fenton, presenta el inconveniente de la producción de fangos y el requerimiento de trabajar a rangos de $\mathrm{pH}$ ácidos, con el consiguiente costo adicional que supone el ajuste del $\mathrm{pH}$ y el acondicionamiento y disposición posterior de los lodos generados (Levchuk et al., 2014; Litter \& Quici, 2010). En la actualidad, se están empleando catalizadores heterogéneos (proceso Fenton heterogéneo) en los que el hierro se fija en la superficie de un soporte (carbón activo, sulfato de aluminio, ślice, zeolitas, etc.) para evitar la formación de fangos, siendo el catalizador recuperable tras finalizar el proceso (Babuponnusami \& Muthukumar, 2014; Parra et al., 2004). Además, el sistema Fenton heterogéneo pueden ser operado a condiciones de $\mathrm{pH}$ cercanas a la neutralidad y asistido con radiación UVvisible (sistema foto-Fenton heterogéneo) (Parra et al., 2004).

Por otro lado, existen diversos reportes encaminados a evaluar la viabilidad de la aplicación del proceso Fenton y foto-Fenton combinados con otros PAO, tales como $\mathrm{UV} / \mathrm{TiO}_{2}$, ultrasonidos y oxidación electroquímica (Babuponnusami \& Muthukumar, 2014; Pimentel et al., 2008; Torres et al., 2007). Si bien, la implementación de estos sistemas híbridos a escala industrial es costosa en comparación con los sistemas biológicos convencionales (Sánchez et al., 2013). Por ello, se plantea la posibilidad de llevar a cabo una oxidación parcial de los compuestos a degradar, con el fin de originar sustancias de escasa toxicidad y mayor biodegradabilidad que pudieran ser mineralizadas en un tratamiento biológico posterior (Rubio-Clemente et al., 2014). De este modo, se minimizarían los costos de operación asociados al empleo de este tipo de tecnologías. No obstante, debe tenerse presente que antes de proceder al acoplamiento del proceso Fenton o foto-Fenton con el sistema biológico, deben de realizarse pruebas de toxicidad del efluente procedente del proceso químico y eliminarse por completo el $\mathrm{H}_{2} \mathrm{O}_{2}$ residual, ya que éste podría inhibir la actividad microbiana (Babuponnusami \& Muthukumar, 2014).

\section{Conclusiones}

Los efluentes procedentes de la industria petroquímica son muy variados y presentan una alta cantidad de compuestos tóxicos, persistentes y difíciles de degradar por procesos convencionales. Por lo tanto, se hace necesario el empleo de tecnologías más eficientes. En este sentido, el proceso de oxidación Fenton puede ser considerado como una técnica atractiva en el tratamiento de este tipo de aguas residuales. Las variables más influyentes en el sistema Fenton son la concentración de $\mathrm{H}_{2} \mathrm{O}_{2} \mathrm{y}$ de $\mathrm{Fe}^{2+}$, la temperatura, el $\mathrm{pH}$, el tiempo de reacción, el contenido y naturaleza de los contaminantes objeto de estudio, así como la presencia de aniones en el afluente a tratar. A pesar de que existen diversos reportes encaminados a determinar los distintos rangos 
de operación de cada uno de estos parámetros, no pueden establecerse unos valores óptimos de funcionamiento, a excepción del $\mathrm{pH}$, ya que están fuertemente influenciados por la composición del afluente. Por este motivo, y debido a que las aguas residuales de origen petroquímico presentan una gran variabilidad, resulta bastante complejo optimizar el proceso Fenton a escala industrial, haciéndose necesarios ensayos previos a escala de laboratorio y a escala piloto.

Aunque el sistema Fenton ha mostrado ser efectivo en el tratamiento de afluentes derivados de la industria del petróleo, este proceso presenta inconvenientes ligados fundamentalmente al uso de grandes cantidades de hierro para conseguir altos rendimientos. En este sentido, el proceso foto-Fenton requiere de menores cantidades de catalizador para obtener buenos resultados. Sin embargo, ambas tecnologías generan fangos y precisan de condiciones ácidas. Estas limitaciones pueden ser superadas mediante la aplicación de los sistemas heterogéneos Fenton y foto-Fenton. Por otro lado, recientes estudios se basan en el empleo de sistemas híbridos en los que se combinan los procesos Fenton y foto-Fenton con otros PAO(UV/ $\mathrm{TiO}_{2}$, ultrasonidos, oxidación electroquímica). No obstante, el principal problema de estas tecnologías de oxidación es el elevado costo que supone su implementación a escala industrial. Por este motivo, el acoplamiento de estos sistemas avanzados de oxidación como pretratamiento a procesos biológicos convencionales se posiciona como una alternativa económicamente viable para la remoción de contaminantes tóxicos y persistentes, como los derivados del petróleo.

\section{Agradecimientos}

La estudiante de doctorado agradece al Instituto Colombiano de Crédito Educativo y Estudios Técnicos en el Exterior (ICETEX) por su apoyo económico.

\section{Referencias}

Babuponnusami, A., \& Muthukumar, K. (2014). A review on Fenton and improvements to the Fenton process for wastewater treatment. Journal of Environmental Chemical Engineering 2 (1), 557-572.

Batista, A.P.S., \& Pupo, R.F. (2012). Parameters affecting sulfonamide photo-Fenton degradationIron complexation and substituent group. Journal of Photochemistry and Photobiology A:Chemistry 232, 8-13.

Bautista, P., Mohedano, A.F., Gilarranz, M.A., Casas, J.A., \& Rodríguez, J.J. (2007). Application of Fenton oxidation to cosmetic wastewaters treatment. Journal of Hazardous Materials 143 (12), 128-134.

Bianco, B., De Michelis, I., \& Vegliò, F. (2011). Fenton treatment of complex industrial wastewater: optimization of process conditions by surface response method. Journal of Hazardous Materials $186(2-3), 1733-1738$.

Burbano, A.A., Dionysiou, D.D., \& Suidan, M.T. (2008). Effect of oxidant-to-substrate ratios on the degradation of MTBE with Fenton reagent. Water Research 42 (12), 3225-3239.

Chamarro, E., Marco, A., \& Esplugas, S., (2001). Use of Fenton reagent to improve organic chemical biodegradability. Water Research 35 (4), 1047-1051.

Coelho, A., Castro, A.V., Dezotti, M., \& Sant'Anna, G.L.Jr. (2006). Treatment of petroleum refinery sourwater by advanced oxidation processes. Journal of Hazardous Materials 137 (1), 178-184.

Da Rocha, O.R.S., Dantas, R.F., Bezerra, M.M.M., Lima, M.M., Lins, V. (2013). Solar photo-Fenton treatment of petroleum extraction wastewater. Desalination and Water Treatment 51 (28-30), 5785-5791.

Domenech, X., Wilson, F., Jardim, W., \& Litter, M. (2004). Procesos avanzados de oxidación para la eliminación de contaminantes. In: M.A. Blesa \& B. Sánchez (editores), Eliminación de contaminantes por fotocatálisis heterogénea. Colección Documentos Ciemat, (Capítulo 1). 
Dopar, M., Kusic, H., \& Koprivanac, N. (2011). Treatment of simulated industrial wastewater by photo-Fenton process. Part I: The optimization of process parameters using design of experiments (DOE). Chemical Engineering Journal 173( 2), 267-279.

Durán-Moreno, A., García-González, S.A., Gutiérrez-Lara, M.R., Rigas, F., \& RamírezZamora, R.M. (2011). Assessment of Fenton's reagent and ozonation as pre-treatments for increasing the biodegradability of aqueous diethanolamine solutions from an oil refinery gas sweetening process. Journal of Hazardous Materials 186, 1652-1659.

Esplugas, S., Giménez, J., Contreras, S., Pascual, E., \& Rodríguez, M. (2002). Comparison of different advanced oxidation processes for phenol degradation. Water Research 36 (4), 1034-1042.

Galvão, S.A., Mota, A.L., Silva, D.N., Moraes, J.E., Nascimento, C.A., \& Chiavone-Filho, O. (2006). Application of the photo-Fenton process to the treatment of wastewaters contaminated with diesel. The Science of the Total Environment 367 (1), 42-49.

Ghosh, P., Samanta, A.N., \& Ray, S. (2010). COD reduction of petrochemical industry wastewater using Fenton's oxidation. The Canadian Journal of Chemical Engineering 88 (6), 1021-1026.

Glaze, W.H., Kang, J.W., \& Chapin, D.H. (1987). The Chemistry of Water Treatment Processes Involving Ozone, Hydrogen Peroxide and Ultraviolet Radiation. Ozone: Science \& Engineering 9 (4), 335-352.

Grčić, I., Vujević, D., Šepčić, J., \& Koprivanac, N. (2009). Minimization of organic content in simulated industrial wastewater by Fenton type processes: A case study. Journal of Hazardous Materials 170 (2-3), 954-961.

Guimarães, J.R., Gasparini, M.C., Maniero, M.G., \& Mendes, C.G.N. (2012). Stripped sour water treatment by advanced oxidation processes.
Journal of the Brazilian Chemical Society 23 (9), 1680 - 1687.

Hasan, D.B., Aziz, A.R.B., \& Daud, W.M.A.W. (2012). Oxidative mineralisation of petroleum refinery effluent using Fenton-like process. Chemical Engineering Research and Design, 90 (2), 298-307.

Homem, V., Dias, Z., Santos, L., \& Alves, A. (2009). Preliminary feasibility study of benzo(a)pyrene oxidative degradation by Fenton treatment. Journal of Environmental and Public Health 2009, 1 - 6.

Hong, S., Zhang, H., Duttweiler, C.M., \& Lemley, A.T. (2007). Degradation of methyl tertiarybutyl ether (MTBE) by anodic Fenton treatment. Journal of Hazardous Materials 144 (1-2), 29-40.

Hu, Q., Zhang, C., Wang, Z., Chen, Y., Mao, K., Zhang, X., Xiong, Y., \& Zhu, M. (2008). Photodegradation of methyl tert-butyl ether (MTBE) by UV/H2O2 and $\mathrm{UV} / \mathrm{TiO}_{2}$. Journal of Hazardous Materials 154 (1-3), 795-803.

Kavitha, V., \& Palanivelu, K. (2004). The role of ferrous ion in Fenton and photo-Fenton processes for the degradation of phenol. Chemosphere 55 (9), 1235-1243.

Levchuk, I., Bhatnagar, A., \& Sillanpää, M. (2014). Overview of technologies for removal of methyl tert-butyl ether (MTBE) from water. Science of The Total Environment 476-477, 415-433.

Litter, M.I., \& Quici, N. (2010). Photochemical advanced oxidation processes for water and wastewater treatment. Recent Patents on Engineering 4, 217-241.

Maciel, R., Sant'Anna, G.L., \& Dezotti, M. (2004). Phenol removal from high salinity effluents using Fenton's reagent and photo-Fenton reactions. Chemosphere 57 (7), 71-719.

Malíková, P., Hajduková, J., \& Nezvalová, L., (2009). Oxidation of polycyckic aromatic hydrocarbons by fenton reaction. GeoScience Engineering LV (4), 23-28. 
Manariotis, I.D., Karapanagioti, H.K., \& Chrysikopoulos, C.V. (2011). Degradation of PAHs by high frequency ultrasound. Water Research, 45 (8), 2587-2594.

Mandal, T., Maity, S., Dasgupta, D., \& Datta, S. (2010). Advanced oxidation process and biotreatment: Their roles in combined industrial wastewater treatment. Desalination 250 (1), 87-94.

Manoli, E., \& Samara, C. (2008). The removal of Polycyclic Aromatic Hydrocarbons in the wastewater treatment process: Experimental calculation and model prediction. Environmenta Pollution 151, 477-485.

Neyens, E., \& Baeyens, J. (2003). A review of classic Fenton's peroxidation as an advanced oxidation technique. Journal of Hazardous Materials 98 (1-3), 33-50.

Nichela, D.A., Berkovic, A.M., Costante, M.R., Juliarena, M.P., \& García, F.S. (2013). Nitrobenzene degradation in Fenton-like systems using $\mathrm{Cu}$ (II) as catalyst. Comparison between $\mathrm{Cu}(\mathrm{II})$ - and $\mathrm{Fe}(\mathrm{III})-$ based systems. Chemical Engineering Journal $228,1148-1157$.

Oller, I., Malato, S., \& Sánchez-Pérez, J.A. (2011). Combination of Advanced Oxidation Processes and biological treatments for wastewater decontamination--A review. The Science of the Total Environment 409 (20) 4141-4166.

Padoley, K.V., Mudliar, S.N., Banerjee, S.K., Deshmukh, S.C., \& Pandey, R.A. (2011). Fenton oxidation: A pretreatment option for improved biological treatment of pyridine and 3-cyanopyridine plant wastewater. Chemical Engineering Journal 166 (1), 1-9.

Parra, S., Henao, L., Mielczarski, E., Mielczarski, J., Albers, P., Suvorova, E., Guindet, J., \& Kiwi, J. (2004). Synthesis, testing, and characterization of a novel Nafion membrane with superior performance in photoassisted immobilized Fenton catalysis. Langmuir 20 (13), 5621-5629.
Patterson, C.L., Cadena, F., Sinha, R., Ngo-Kidd, D.K., Ghassemi, A., \& Krishnan, E.R. (2013). Field Treatment of MTBE Contaminated Groundwater Using Ozone/UV Oxidation. Groundwater Monitoring \& Remediation 33 (2), 44-52.

Pignatello, J.J., Oliveros, E., \& MacKay, A. (2006). Advanced Oxidation Processes for Organic Contaminant Destruction Based on the Fenton Reaction and Related Chemistry. Critical Reviews in Environmental Science and Technology 36 (1), 1-84.

Pimentel, M., Oturan, N., Dezotti, M., \& Oturan, M.A. (2008). Phenol degradation by advanced electrochemical oxidation process electro-Fenton using a carbon felt cathode. Applied Catalysis B: Environmental 83(1-2), 140-149.

Pontes, R.F., Moraes, J.E., Machulek, A.Jr., \& Pinto, J.M. (2010). A mechanistic kinetic model for phenol degradation by the Fenton process. Journal of Hazardous Materials 176 (1-3), 402-413.

Qiao, M., Qi, W., Liu, H., \& Qua, J. (2014). Occurrence, behavior and removal of typical substituted and parent polycyclic aromatic hydrocarbons in a biological wastewater treatment plant. Water Research 52, 11-19.

Rubio-Clemente, A., Torres-Palma, R.A., \& Peñuela, G.A. (2014). Removal of polycyclic aromatic hydrocarbons in aqueous environment by chemical treatments: A review. Sciences of The Total Environment 478, 201-225.

Sánchez, J.A., Román, I.M., Carra, I., Cabrera, A., Casas, J.L., \& Malato, S. (2013). Economic evaluation of a combined photo-Fenton/MBR process using pesticides as model pollutant. Factors affecting costs. Journal of Hazardous Materials 244-245, 195-203.

Siedlecka, E.M., \& Stepnowski, P. (2006a). Decomposition rates of methyl tert-butyl ether and its by-products by Fenton system in saline wastewaters. Separation and Purification Technology 52, 317-324. 
Siedlecka, E.M., \& Stepnowski, P. (2006b). Treatment of oily port wastewater effluents using the ultraviolet/hydrogen peroxide photodecomposition system. Water Environment Research 78 (8), 852-856.

Torres, R.A., Pétrier, C., Combet, E., Moulet, F., \& Pulgarin, C. (2007). Bisphenol A mineralization by integrated ultrasound-UV-iron (II) treatment. Environmental Science and Technology 41 (1), 297-302.

Xu, X.R., Zhao, Z.Y., Li, X.Y., \& Gu, J.D. (2004). Chemical oxidative degradation of methyl tert- butyl ether in aqueous solution by Fenton's reagent. Chemosphere 55, 73-79.

Zazo, J.A., Casas, J.A., Mohedano, A.F., \& Rodriguez, J.J. (2009). Semicontinuous Fenton oxidation of phenol in aqueous solution. A kinetic study. Water Research 43 (16), 4063-4069.

Zazo, J.A., Casas, J.A., Mohedano, A.F., Gilarranz, M.A., \& Rodríguez, J.J. (2005). Chemical pathway and kinetics of phenol oxidation by Fenton's reagent. Environmental Sciences \& Technology 39, 9295-9302. 